\title{
Adaptive All-Optical Ultrasound Imaging through Temporal Modulation of Excitation Light
}

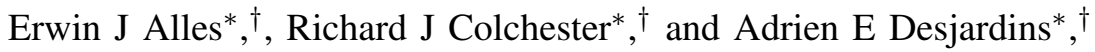 \\ *Department of Medical Physics \& Biomedical Engineering, University College London, London, United Kingdom \\ ${ }^{\dagger}$ Wellcome / EPSRC Centre for Surgical and Interventional Sciences, University College London, London, United Kingdom \\ E-mail: e.alles@ucl.ac.uk
}

\begin{abstract}
In this work, we demonstrate how the wide acoustic bandwidths generated by all-optical ultrasound imaging probes can be leveraged to dynamically trade-off image resolution with penetration depth. This dynamic trade-off was achieved through temporal modulation of the excitation light using bandwidthlimited linear chirps. The penetration depth of the all-optical ultrasound imaging probe used here could be arbitrarily set between 2 and $15 \mathrm{~mm}$, to achieve inversely proportional spatial resolutions ranging from $128-500 \mu \mathrm{m}$ (lateral) and $189-295 \mu \mathrm{m}$ (axial). This added versatility in image parameters will enable seamless multi-scale ultrasound imaging that can strongly benefit interventional imaging.
\end{abstract}

Index Terms-All-optical ultrasound, chirp modulation, resolution, penetration depth, multi-scale imaging

\section{INTRODUCTION}

Conventional electronic ultrasound transducers typically derive their sensitivity from mechanical resonance. As such, their centre frequencies and bandwidths are fixed during manufacturing. As the ultrasound bandwidth determines the image resolution, and frequency-dependent attenuation limits the imaging depth [1], electronic ultrasound imaging probes are typically tailored to specific anatomies and pathologies. Consequently, typical ultrasound clinics employ a range of different ultrasound imaging probes to accommodate a variety of imaging contexts.

Recently, all-optical ultrasound imaging has been demonstrated as a viable alternative to electronic technology. With all-optical ultrasound imaging, ultrasound is both generated and detected using light: ultrasound is generated optically within an optically absorbing structure via the photoacoustic effect [2], and optically resonant structures [3]-[8] are employed to measure the minute deformations caused by impinging back-scattered acoustic waves. Using only fibreoptic technology and miniature optics, all-optical ultrasound imaging probes can be readily miniaturised, can be inherently insensitive to electromagnetic radiation (and hence compatible with MRI), and can be relatively inexpensive. Hence, they are ideally suited to biomedical and especially interventional applications. In recent years, all-optical ultrasound imaging

This work was supported by an ERC Starting Grant [310970 MOPHIM], the EU project FAMOS [FP7 ICT, Contract 317744], the Innovative Engineering for Health award by the Wellcome Trust [WT101957] and the EPSRC [NS/A000027/1], the EPSRC Healthcare Technologies Challenge Award [EP/N021177/1], and the EPSRC Doctoral Training Partnership [EP/N509577/1]. of biological tissue has been demonstrated using various imaging paradigms [3]-[6], [9]-[14], and the first in vivo application [15] and video-rate bench-top imaging system [16] have been reported.

Optical ultrasound sources typically employ Q-switched lasers emitting light pulses with durations of a few nanoseconds, corresponding to optical bandwidths exceeding $100 \mathrm{MHz}$. This light is converted into ultrasound in an optically absorbing structure; to date the most efficient optical ultrasound generators reported used nanocomposites comprising functionalised multi-walled carbon nanotubes (MWCNTs) [13], [17] or candle soot particles [18] as optical absorbers. These optical absorbers were embedded in polydimethylsiloxane (PDMS) acting as an elastomeric host with a high thermal expansion coefficient. Such nanocomposite structures are capable of generating high pressures (exceeding $4 \mathrm{MPa}$ at a distance of $1.5 \mathrm{~mm}$ for an optical ultrasound source with a diameter of just $200 \mu \mathrm{m}$ [13]) that rival those generated using electronic transducers, at wide acoustic bandwidths of $30-40 \mathrm{MHz}$.

While typical optical ultrasound sources generate very wide bandwidths, they are not well matched to the bandwidth of the optical excitation. Hence, a significant part of the optical energy does not contribute to the optical ultrasound generation, and it has previously been shown [19] that matching the optical to the acoustical bandwidth significantly improves the transduction efficiency. As a result, less powerful laser diodes (that tend to be cheaper and more compact than Q-switched lasers) could be used to obtain the same image contrast and resolution. In this work, in addition to bandwidth matching, we deliberately limited the bandwidth of the optical excitation (and hence of the generated ultrasound) in order to achieve dynamic control over the trade-off of the penetration depth and imaging resolution of all-optical ultrasound. By imaging resolution phantoms and tissue samples, we demonstrated how, using a single imaging probe and aperture, temporally modulated all-optical ultrasound enables true multi-scale ultrasound imaging.

\section{Methods}

\section{A. Optical ultrasound detection}

Back-scattered ultrasound waves were detected using a fibreoptic detector comprising a Fabry-Pérot cavity on its tip. This miniature detector (diameter: $125 \mu \mathrm{m}$ ) achieved a very high 
sensitivity (noise-equivalent pressure: $40 \mathrm{~Pa}$ ), a wide bandwidth (up to $80 \mathrm{MHz}$ ), and nearly omni-directional response, while at the same time being resilient to electromagnetic interference and MRI-compatible [20]. This fibre-optic detector consisted of a set of dielectric mirrors separated by a polymer spacer, and derived its sensitivity to impinging ultrasound waves from shifts in the optical resonance wavelength due to the associated thickness change. As a result, an impinging ultrasound wave modulated the amplitude of continuouswave interrogation light reflected off the cavity [21], which was detected using a photodiode and digitiser (M4i.4420-x8, Spectrum, Germany).

\section{B. Optical ultrasound generation}

Ultrasound waves were optically generated within a MWCNTPDMS nanocomposite coating [13] deposited on the tip of an optical fibre (diameter: $200 \mu \mathrm{m}$ ). Excitation light (either pulsed or temporally modulated) was delivered to this coating, where it was converted into ultrasound via the photoacoustic effect. This fibre-optic ultrasound source was paired with the fibreoptic ultrasound detector to obtain a miniature, single-element all-optical ultrasound imaging probe. The frequency response of this all-optical imaging probe was determined from pulseecho measurements obtained from a single tungsten wire (diameter: $27 \mu \mathrm{m}$ ) placed directly in front of the probe at a distance of $3.1 \mathrm{~mm}$.

\section{Optical excitation}

Either pulsed or temporally modulated excitation light was delivered to the optical ultrasound genering coating.

Pulsed: A Q-switched laser (wavelength: $1064 \mathrm{~nm}$, repetition rate: $100 \mathrm{~Hz}$; SPOT-10-500-1064, Elforlight, U.K.) was used to deliver light pulses (pulse duration: 2 ns, pulse energy: $15 \mu \mathrm{J}$ ) with sufficient energy to allow for pulse-echo data acquisition without the need for signal averaging.

Temporally modulated: A high-power laser diode (power: $500 \mathrm{~mW}$; LU1064M450, Lumics, Germany) driven by highbandwidth driver (bandwidth: $400 \mathrm{MHz}$; BFS-VRM-03-HP, Picolas, Germany) was used to generate temporally modulated optical excitations. An arbitrary waveform generator (33611A, Agilent, CA, USA) sampled at $200 \mathrm{MHz}$ was used to generate linear chirps of the form

$$
c(t)=\sin \left(2 \pi\left[f_{\min } t+\frac{f_{\max }-f_{\min }}{2 \delta} t^{2}\right]\right)+1,
$$

where $0 \leq t \leq \delta$, chirp duration $\delta=4 \mu$ s, and the minimum and maximum frequencies $f_{\min }$ and $f_{\max }$ can be arbitrarily chosen. This chirp signal was offset to ensure $c(t) \geq 0$, thus ensuring a positive optical power, and a Tukey taper was used to suppress ultrasound signals arising from discontinuous edges of the chirp signal [19]. These chirps were generated at a repetition rate of $900 \mathrm{~Hz}$ and a pulse energy of $1.6 \mu \mathrm{J}$. To achieve a similar signal-to-noise ratio (SNR) despite a reduced pulse energy, 100-fold averaging was applied.
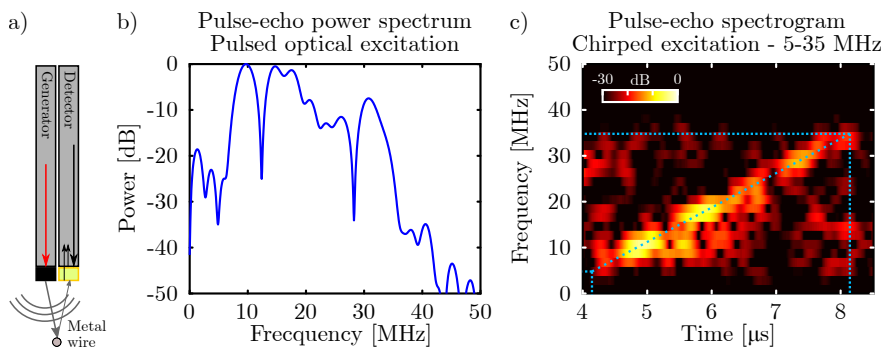

Fig. 1. Frequency response of the all-optical ultrasound imaging probe. (a) A fibre-optic ultrasound generator was paired with a fibre-optic ultrasound detector to obtain a single-element, all-optical ultrasound imaging probe. The back-scattered reflection off a metal wire was used to measure the frequency response of the imaging probe. (b) Normalised power spectrum of the pulseecho signal obtained using nanosecond-duration pulsed optical excitation. (c) Spectrogram of the pulse-echo signal obtained using a linear chirp (duration: $4 \mu \mathrm{s}$, bandwidth: $5-35 \mathrm{MHz}$ ) as optical excitation. The blue dotted lines indicate the frequency limits and the shape of the transmitted linear chirp.

\section{All-optical ultrasound imaging}

All-optical ultrasound imaging was performed by mechanically scanning the single-element imaging probe across a linear aperture whilst continuously recording pulse-echo A-scans. This way, a $15 \mathrm{~mm}$ wide aperture was scanned at a step size of $50 \mu \mathrm{m}$ (MTS50/M-Z8 + TDC001, Thorlabs, Germany). The recorded B-scan data were reconstructed into images through back-projection using the $k$-Wave toolbox [22], and data acquired using chirped excitation light were pulse-compressed prior to reconstruction by cross-correlating each A-scan with chirp $c(t)$. Power-law time-gain compensation (of empirically determined power) was applied to account for propagation and attenuation losses, and envelope detection was applied after image reconstruction.

\section{RESULTS}

\section{A. Frequency response of the imaging probe}

The power spectrum for a pulse-echo signal off a metal wire (Fig. 1(b)) shows that the all-optical ultrasound imaging probe is predominantly sensitive to frequencies between 2 and $35 \mathrm{MHz}$. As the fibre-optic ultrasound detector exhibits a much wider bandwidth [8], this bandwidth limitation is predominantly due to the optical ultrasound generator. Given the very wide bandwidth $(>100 \mathrm{MHz}$ ) of the optical excitation, this result confirms that a significant fraction of the optical energy does not contribute to the optical ultrasound generation. In addition, two sharp dips at approximately 13 and $28 \mathrm{MHz}$ are observed that are due to acoustic defraction around the optical ultrasound detector [8]. The spectrogram obtained using a linear chirp as optical excitation (Fig. 1(c)) confirms these observations, and in addition highlights the reduced SNR due to the lower pulse energy and peak optical power.

\section{B. Image resolution}

Through variation of the optical (and hence acoustical) bandwidth, images were obtained of a wire phantom at a range of resolutions (Fig. 2). Using nanosecond duration pulsed excitation light, a lateral resolution of $398 \mu \mathrm{m}$ and axial 

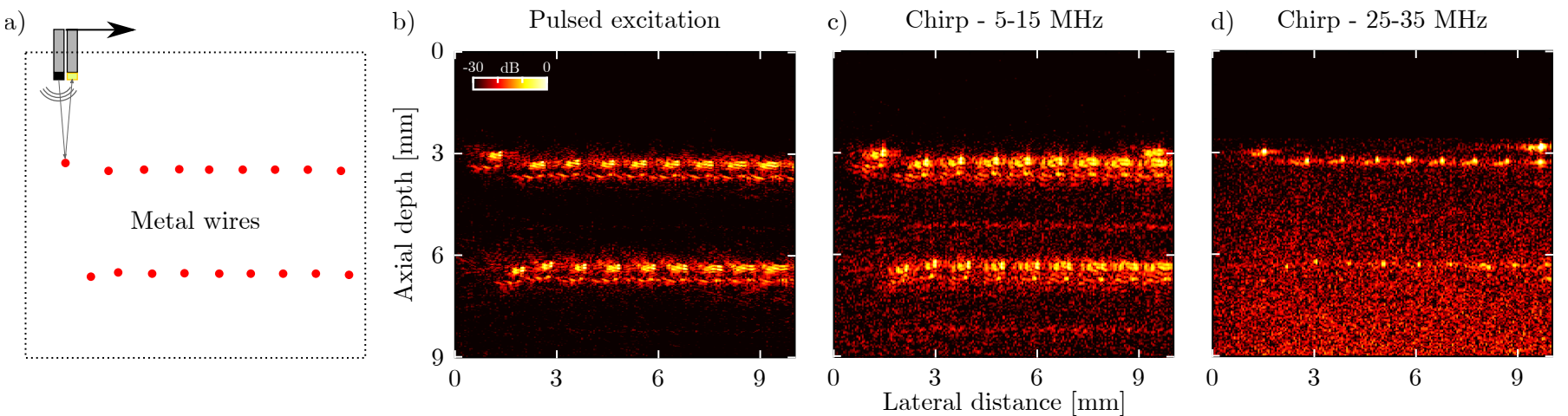

Fig. 2. Image resolution versus bandwidth. (a) Schematic of the phantom and imaging paradigm. Two rows of metal wires, placed perpendicular to the image plane, are located at depths of 3.1 and $6.1 \mathrm{~mm}$. The wires were spaced $1 \mathrm{~mm}$ apart. (b) All-optical ultrasound image obtained using nanosecond duration pulsed optical excitation. (c) All-optical ultrasound image obtained using chirped optical excitation using only low frequencies between 5 and $15 \mathrm{MHz}$. (d) All-optical ultrasound image obtained using chirped optical excitation using only high frequencies between 25 and 35 MHz. All images are shown on independently normalised logarithmic scales.

resolution of $192 \mu \mathrm{m}$ were obtained (Fig. 2(b)). By confining the bandwidth of the excitation light using linear chirp optical excitation (Fig. 2(c)), low-frequency (5-15 MHz) images yielded lateral and axial resolutions of $500 \mu \mathrm{m}$ and $295 \mu \mathrm{m}$, respectively. Using only the high frequencies $(25-35 \mathrm{MHz})$, lateral and axial resolutions of $128 \mu \mathrm{m}$ and $189 \mu \mathrm{m}$ were obtained (Fig. 2(d)). However, as the all-optical ultrasound imaging probe was most sensitive between 8 and $20 \mathrm{MHz}$ (Fig. 1(b)), the contrast for images obtained using frequencies outside this range was decreased, as is confirmed in Fig. 2(d). The "echoes" observed approximately $0.5 \mathrm{~mm}$ below each wire were due to ringing within a section of tubing used to mount the fibre-optic source and receiver.

\section{Penetration depth}

The impact of the acoustical bandwidth on the penetration depth is highlighted in Fig. 3, where a section of chicken breast was imaged using both pulsed and temporally modulated excitation light. Using pulsed excitation light, an imaging depth of up to $12 \mathrm{~mm}$ was achieved, with information mainly located around the boundaries of the tissue sample (Fig. 3(b)). In contrast, using low-frequency modulated excitation light $(2-12 \mathrm{MHz})$, signal is observed throughout the entire sample image, but at lower resolution (Fig. 3(c)). An imaging depth of $15 \mathrm{~mm}$ was achieved, although this was limited here by the temporal acquisition window for each A-scan; larger depths are expected to be feasible. Some artefacts can be observed above the tissue sample; these are caused by the lower A-scan SNR and pulse compression side lobes. When excitation light containing only higher frequencies $(25-35 \mathrm{MHz})$ is used, the resolution is improved, at the expense of a reduced imaging depth of just $2 \mathrm{~mm}$ (Fig. 3(d)). Note that this impact of the frequency on the penetration depth is much less pronounced in the images of the wire phantom (Fig. 2) as the acoustical attenuation in water is much weaker than in chicken breast tissue.

\section{Discussion AND CONCLUSION}

In this work, a novel optical excitation paradigm for alloptical ultrasound imaging is presented. While traditionally nanosecond duration light pulses are used, here we employ temporally modulated excitation light generated by a highpower, high-bandwidth laser diode. Using bandwidth-limited linear chirps, the optical (and hence acoustical) bandwidth of the system can be adjusted to trade-off penetration depth with image resolution, as demonstrated here on imaging phantoms.

In addition, the ability to constrain the optical bandwidth was used to improve the optical-to-ultrasound transduction efficiency by avoiding those frequencies for which the imaging probe has low sensitivity, as was previously demonstrated in [19]. This allowed for a small laser diode to be used, despite generating a peak optical power that is five orders of magnitude lower than that of conventional Q-switched laser sources. In this work, this difference in peak power was offset by temporally extending the chirp to the microsecond range, and in addition applying 100-fold signal averaging. Despite these efforts, the images acquired using bandwidth-constrained optical excitations were of lower contrast than those obtained using pulsed excitation. However, coded excitation schemes (e.g. Barker or Golay pairs) could be used to further average out noise, and a fibre laser amplifier could be added to boost the A-scan SNR.

In principle, the bandwidth limitations implemented here could be performed in post-processing using band-pass filters. However, this would result in energy loss and severely reduce the SNR of the data, thus further reducing the transduction efficiency. By limiting the bandwidth of the optical excitation instead, a larger fraction of the transmitted optical energy contributes to the ultrasound image. Consequently, the energy deposition within the optical ultrasound generating coating is limited, which ultimately allows for higher pulse repetition rates and image frame rates.

The wide range of tuneability of the imaging resolution and penetration demonstrated here will allow for seamless multiscale imaging using a single imaging probe, and obviate the 

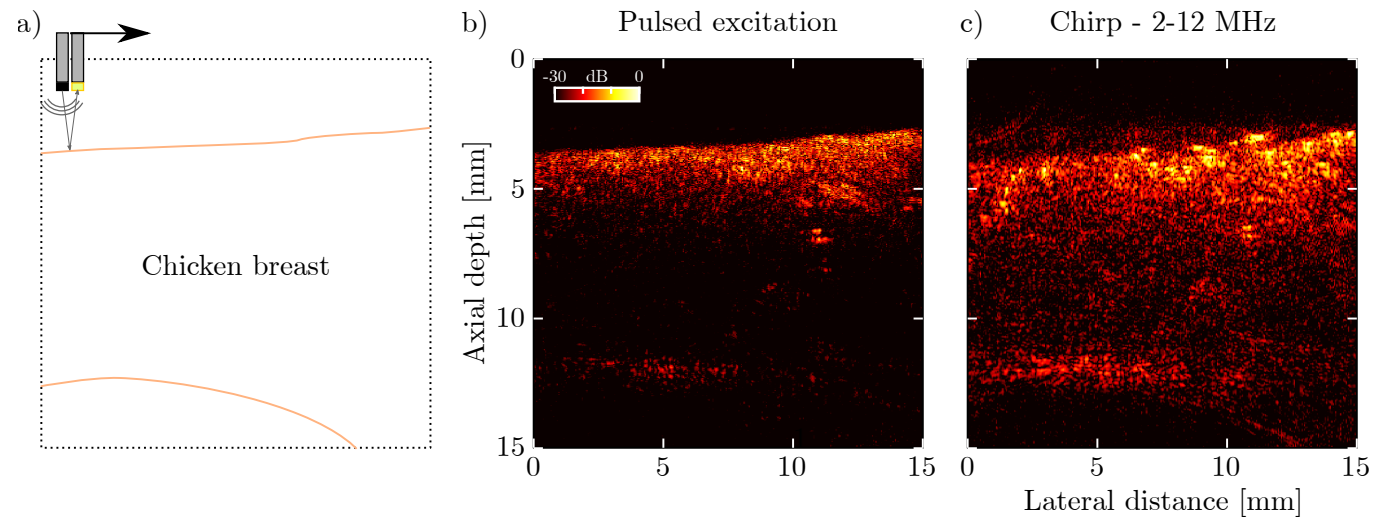

Fig. 3. Penetration depth versus bandwidth. (a) Schematic of the phantom and imaging paradigm. A thick section of chicken breast (of a spatially varying thickness between 9 and $19 \mathrm{~mm}$ ) was mounted and imaged. (b) All-optical ultrasound image obtained using nanosecond duration pulsed optical excitation. (c) All-optical ultrasound image obtained using chirped optical excitation using only low frequencies between 2 and 12 MHz. (d) All-optical ultrasound image obtained using chirped optical excitation using only high frequencies between 25 and $35 \mathrm{MHz}$. All images are shown on independently normalised logarithmic scales.

need for multiple imaging probes to be used. This will greatly facilitate multi-scale imaging, and result in reduced procedure times, especially in an interventional setting.

\section{REFERENCES}

[1] RSC Cobbold. Foundations of Biomedical Ultrasound. Oxford University Press, USA, New York, 2007.

[2] PC Beard. Biomedical photoacoustic imaging. Interface Focus, 1(4):602-631, 2011.

[3] Y Hou, J-S Kim, S-W Huang, S Ashkenazi, L Jay Guo, and M O'Donnell. Characterization of a broadband all-optical ultrasound transducer-from optical and acoustical properties to imaging. Ultrasonics, Ferroelectrics, and Frequency Control, IEEE Transactions on, 55(8): 1867-1877, 2008

[4] B-Y Hsieh, S-L Chen, T Ling, LJ Guo, and P-C Li. All-optical scanhead for ultrasound and photoacoustic imaging - imaging mode switching by dichroic filtering. Photoacoustics, 2(1):39-46, 2014.

[5] E Biagi, S Cerbai, L Masotti, L Belsito, A Roncaglia, G Masetti, and N Speciale. Fiber optic broadband ultrasonic probe for virtual biopsy: Technological solutions. Journal of Sensors, 2010, 2010.

[6] C Sheaff and S Ashkenazi. Polyimide-etalon all-optical ultrasound transducer for high frequency applications. In Proceedings SPIE BiOS, pages 89434M-89434M-8. International Society for Optics and Photonics, 2014.

[7] SM Leinders, WJ Westerveld, J Pozo, PLMJ van Neer, B Snyder, P O'Brien, HP Urbach, N de Jong, and MD Verweij. A sensitive optical micro-machined ultrasound sensor (OMUS) based on a silicon photonic ring resonator on an acoustical membrane. Scientific Reports, 5, 2015.

[8] JA Guggenheim, J Li, TJ Allen, RJ Colchester, S Noimark, OO Ogunlade, IP Parkin, I Papakonstantinou, AE Desjardins, EZ Zhang, and PC Beard. Ultrasensitive plano-concave optical microresonators for ultrasound sensing. Nature Photonics, 11(11):714-719, 2017.

[9] Y Hou, S Ashkenazi, S-W Huang, and M O'Donnell. An integrated optoacoustic transducer combining etalon and black PDMS structures. Ultrasonics, Ferroelectrics, and Frequency Control, IEEE Transactions on, 55(12):2719-2725, 2008.

[10] C Sheaff and S Ashkenazi. An all-optical thin-film high-frequency ultrasound transducer. In Proceedings IEEE IUS, pages 1944-1947. IEEE, 2011.

[11] RJ Colchester, EZ Zhang, CA Mosse, PC Beard, I Papakonstantinou, and AE Desjardins. Broadband miniature optical ultrasound probe for high resolution vascular tissue imaging. Biomedical Optics Express, 6(4):1502-1511, 2015.

[12] EJ Alles, S Noimark, E Zhang, PC Beard, and AE Desjardins. Pencil beam all-optical ultrasound imaging. Biomedical Optics Express, 7(9):3696-3704, 2016.
[13] S Noimark, RJ Colchester, BJ Blackburn, EZ Zhang, EJ Alles, S Ourselin, PC Beard, I Papakonstantinou, IP Parkin, and AE Desjardins. Carbon-nanotube-PDMS composite coatings on optical fibres for alloptical ultrasound imaging. Advanced Functional Materials, 26(35), 2016.

[14] JL Johnson, J Shragge, and K van Wijk. Nonconfocal all-optical laserultrasound and photoacoustic imaging system for angle-dependent deep tissue imaging. Journal of Biomedical Optics, 22(4):041014-041014, 2017.

[15] MC Finlay, CA Mosse, RJ Colchester, S Noimark, EZ Zhang, S Ourselin, PC Beard, RJ Schilling, IP Parkin, I Papakonstantinou, and AE Desjardins. Through-needle all-optical ultrasound imaging in vivo: a preclinical swine study. Light: Science \& Applications, 6(12):e17103, 2017.

[16] EJ Alles, S Noimark, E Maneas, EZ Zhang, IP Parkin, PC Beard, and AE Desjardins. Video-rate all-optical ultrasound imaging. Biomedical Optics Express, 9(8):3481-3494, 2018.

[17] S Noimark, RJ Colchester, RK Poduval, E Maneas, EJ Alles, T Zhao, EZ Zhang, M Ashworth, E Tsolaki, and AH Chester. Polydimethylsiloxane composites for optical ultrasound generation and multimodality imaging. Advanced Functional Materials, 28(9), 2018.

[18] W-Y Chang, XA Zhang, J Kim, W Huang, A Bagal, C-H Chang, $\mathrm{T}$ Fang, HF Wu, and $\mathrm{X}$ Jiang. Evaluation of photoacoustic transduction efficiency of candle soot nanocomposite transmitters. IEEE Transactions on Nanotechnology, 2018.

[19] EJ Alles, RJ Colchester, and AE Desjardins. Adaptive light modulation for improved resolution and efficiency in all-optical pulse-echo ultrasound. Ultrasonics, Ferroelectrics, and Frequency Control, IEEE Transactions on, 63(1):83-90, 2016.

[20] EZ Zhang and PC Beard. Characteristics of optimized fibre-optic ultrasound receivers for minimally invasive photoacoustic detection. In Proceedings SPIE BiOS, pages 932311-932311-9. International Society for Optics and Photonics, 2015.

[21] E Zhang, JG Laufer, and PC Beard. Backward-mode multiwavelength photoacoustic scanner using a planar Fabry-Pérot polymer film ultrasound sensor for high-resolution three-dimensional imaging of biological tissues. Applied Optics, 47(4):561-577, 2008.

[22] BE Treeby and BT Cox. k-Wave: Matlab toolbox for the simulation and reconstruction of photoacoustic wave fields. Journal of biomedical optics, 15(2):021314-021314-12, 2010. 